\title{
Anoxybiotische Zellvermehrung und Wirkungsmodus der aeroben Wachstumsreaktivierung
}

\author{
Von F. Windisch, W. Heumann und Chr. Goslich \\ Aus dem Institut für Medizin und Biologie der Deutschen Akademie der Wissenschaften zu \\ Berlin (Direktor: Prof. Dr. W. Friedrich), Abteilung Krebsforschung/Mikrobiologie (Prof. \\ Dr. F. W indis c h) \\ (Z. Naturforschg. 8 b, 305-311 [1953]; eingegangen am 8. April 1953)
}

\begin{abstract}
Bei anoxybiotischer Passagenzüchtung nimmt die Zellvermehrung der Gärhefen von Generation zu Generation successiv ab, bis vollständige Cytostase eintritt. Die Kontinuität des Hefewachstums ist obligat aerob bedingt.

Aus dem partiellen und bei fortgesetzter anaerober Passagenimpfung schließlich stagnierenden Proliferationsvermögen der Hefezellen wird — in Analogie zum Muskel — auf das Vorhandensein einer oxydativ gebildeten Energiereserve geschlossen.

Der aerobe Reaktivierungsprozeß bei anoxybiotisch hervorgerufener Wachstumssistierung wird nicht durch einen initialen $\mathrm{O}_{2}$-Effekt ausgelöst, sondern ist zeitlich abhängig von dem in Reaktion tretenden Sauerstoff, der seinerseits in quantitativer Relation zur proliferierenden Zellmasse steht.
\end{abstract}

P a s te u ${ }^{1}$ stellte fest, daß sich junge, lebenskräftige, gesunde Hefezellen auch ohne die Anwesenheit von freiem Sauerstoff (hors de toute présence du gaz oxygène) fortzupflanzen vermögen, dagegen, älter geworden, nur noch mühselig (beaucoup de peine), in einer bizarren und monströsen Form (sous une forme bizarre et monstreuse), zur Vermehrung fähig sind und, noch weiter gealtert, gänzlich zu wachsen aufhören (elles restent absolument inertes dans un milieu dépourvu d'oxygène libre). Wenn nicht auf irgendeine Art wieder Luft hinzutritt, so führt $\mathrm{P}$ a s t e u r weiter aus, erschöpft sich die Lebenskraft der Zellen mehr und mehr (l'activité vitale s'épuise de plus en plus), und die Gärung kann sogar vollständig aufhören (la fermentation peut même s'éteindre).

Beim Wiederaufgreifen des Anaerobiose-Problems vor 25 Jahren konnte Windisch ${ }^{2}$, durch Anwendung einer streng anoxybiotischen Züchtungsmethodik. (Pyrogalloltest), die Beobachtungen P a steurs auf präzisierter experimenteller Grundlage dahingehend bestätigen, daß ,die Hefe bei nicht zu geringer Aussaat in mehreren Generationen völlig anaerob zu sprossen vermag“. Von einer vitalen $\mathrm{Ab}$ schwächung bzw. Degeneration der Organismen, wie von $\mathrm{P}$ asteur angegeben, war indessen nichts $\mathrm{zu}$ bemerken, was sich daraus erklärt, daß die spätere

1 L. P a s t e u r, Etudes sur la bière. Paris 1876.

2 F. W ind is c h, Hoppe-Seyler's Z. physiol. Chem. 179, 88 [1928]. anaerobe Züchtungsweise, abweichend von Pasteurs stationärer Anordnung, bereits periodisch in Passagen erfolgte, wobei jedesmal wieder in ein frisches $\mathrm{O}_{2^{-}}$ freies Nährsubstrat (Würze) übergeimpft wurde; auf diese Art behielten die stets optimal mit Nährstoff versorgten Zellen für lange Zeit ihre unbeeinträchtigte biologische Beschaffenheit bei.

Im gleichen Zusammenhang und im Hinblick auf die nachfolgenden Untersuchungen ist noch ein weiterer Befund, den wir seinerzeit aufzeichneten, erwähnens- und bemerkenswert. Wir stellten schon damals klar heraus, daß die anaerob weitergezüchtete Hefe „zu einem gewissen Zeitpunkt schließlich doch wieder des Sauerstoffs bedarf, um wachstumsfähig zu bleiben“. Dieses anoxybiotische Ergebnis, welches grundsätzlich die Frage nach der Bedeutung des Sauerstoffs für das Zellwachstum aufwirft, greift bereits über die Problemstellung $\mathrm{P}$ as teurs hinaus.

Alle bisherigen Feststellungen in dieser Richtung ${ }^{3}$ beruhen jedoch auf eudiometrischen Bestimmungen, bei denen Quecksilber, in direkter Berührung mit dem biologischen Ansatz, als Sperrflüssigkeit verwendet wird. Es war deshalb erforderlich, im ersten Teil der Arbeit zunächst noch einmal zu überprüfen, ob die vegetative Anabiose auch unter vollständig normalen physiologischen Bedingungen (d.h. im vorliegenden Falle also ohne die Verwendung von Quecksilber als Sperrflüssigkeit) mit gleicher Eindeutigkeit in Erschei-

3 F. W in dis ch, Biochem. Z. 246, 332 [1932]. 
nung tritt. Die Möglichkeit hierzu ergab sich in jüngster Zeit durch Kombination mit der elektrochemischen Oxygenometrie nach $\mathrm{Tödt} \mathrm{t}^{4}$, deren Zuverlässigkeit für mikroquantitative $\mathrm{O}_{2}$-Messungen $(\gamma$-Werte) im biologischen Medium von uns nachgewiesen werden konnte $^{5}$. Im zweiten Teil der Arbeit haben wir Aufklärung darüber zu erhalten versucht, ob im Zustand der Anabiose, hervorgerufen durch Anoxy, der erneut hinzutretende Sauerstoff mit initialem Effekt die Zellvermehrung reaktiviert oder in quantitativer Relation zur proliferierenden Zellmasse steht.

\section{Apparative Methodik}

Für die anoxybiotischen Züchtungsversuche verwendeten wir spezielle, mit vakuumdichten Normalschliffhähnen versehene Kultivierungsgefäße, die derart konstruiert sind, daß jederzeit anaerobe Abimpfungen aus ihnen vorgenommen und laufend elektrochemische Messungen zur Kontrolle der konstanten Anoxy im Reaktionsmedium durchgeführt werden können. Die apparativen Einzelheiten sind aus Abb. $1 \mathrm{zu}$ entnehmen.

Gefäß A dient der anaeroben Züchtung und Überimpfung von Mikroorganismen. Gefäß B, mit einem auswechselbaren Kaliapparat versehen, gestattet außerdem die periodische Bestimmung der im Stoffwechselproze $\beta$ entwickelten $\mathrm{CO}_{2}$ - Menge. Gefäß $\mathrm{C}$ stellt eine für anaerobe Gewebezüchtungen auf fester Plasmaunterlage entsprechend modifizierte Carrelschale dar, die in gleicher Weise wie die anderen Gefäße mit sauerstofffreier Nährlösung beschickt werden kann. Durch Aufsetzen eines mit Schliff versehenen Kaliapparates läßt sich die Kohlensäure, die bei der Atmung entsteht bzw. bei der Glykolyse aus Bicarbonat freigesetzt wird, quantitativ erfassen. Gefäß D, das als elektrochemisches $\mathrm{O}_{2}$-Prüfgerät nach $\mathrm{Tödt}$ ausgebildet ist, ermöglicht den quantitativen Nachweis geringster $\mathrm{O}_{2}$-Mengen (m $\gamma$-Werte) im Nährsubstrat. Für die Vorprüfung der desoxygenisierten Gefäße auf vollständige Luftdichtigkeit an allen Verbindungs- und Schliffstellen hat sich der bereits früher von uns angewandte Pyrogalloltest (farblose Pyrogallollösung, erhalten durch Entlüften der Komponenten vor dem Vermischen) als hochempfindlicher $\mathrm{O}_{2}$-Indikator bewährt *

Bei Testen von grundsätzlicher Entscheidung leiteten wir den nach $\mathrm{H}$ a b e r desoxygenisierten Stickstoff zusätzlich durch eine $\mathrm{CrCl}_{2}$-Lösung, wodurch der $\mathrm{O}_{2}$-Spurengehalt nach den neuesten Ultramessungen von G. Teske (Elektrochem. Abt. Prof. Tödt) bis auf $\mathrm{m} \gamma$-Werte im Liter herabgesetzt wird. Ferner trafen wir noch die besonderen Schutzvorkehrungen, daß wir statt der Normalschliff-Vakuumhähne solche mit Quecksilberdichtung verwendeten und außerdem die anaeroben Kulturen während der Versuchszeit in Gasgefäßen- die mit $\mathrm{O}_{2}$-freiem Stickstoff angefüllt waren, vakuumdicht abkapselten. Auf

4 F. T ö d t, Z. Elektrochem. 54, 485 [1950]; F. T ö d t, S. Kahan u. W. S chwarz, ebenda 56, 19 [1952].

5 Gemeinschaftsarbeit von F. Tödt, G. T e sk e sowie von F. Windisch, W. Heumann u. Chr. Goslich, Biochem. Z. 323, 192 [1952]. diese Weise war es vollkommen unmöglich gemacht, daß auch nur die geringsten Sauerstoffspuren von außen in die Kulturgefäße hineindiffundieren konnten.

\section{Systematik des Arbeits ganges}

Die Abwicklung der anaeroben Vorkehrungen und Maßnahmen gestaltet sich im einzelnen folgendermaßen (siehe

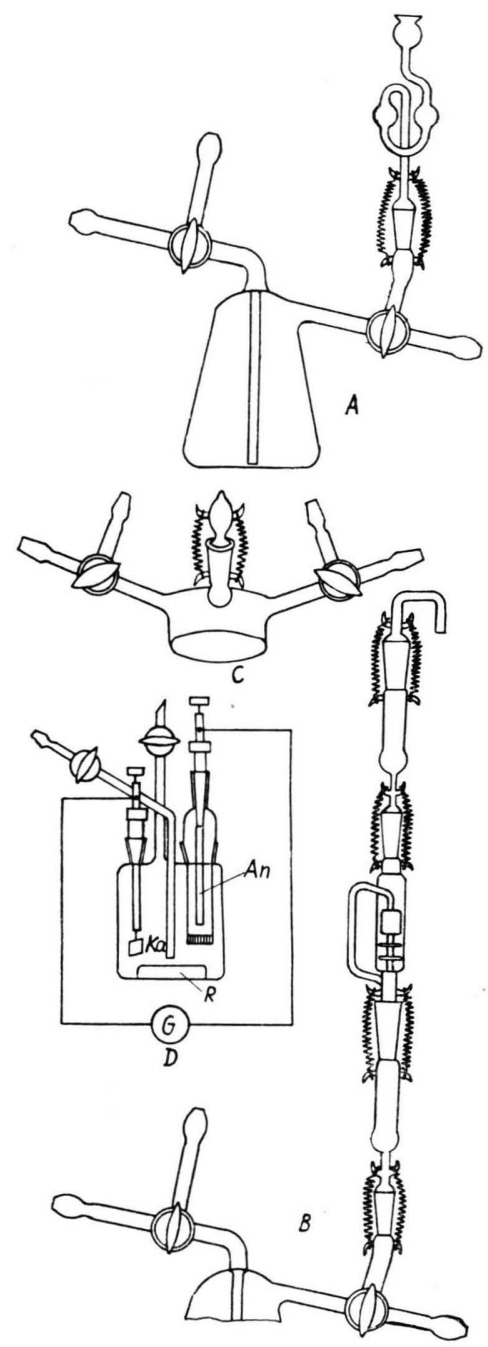

Abb. 1. Züchtungs-, Meß- und Kontrollgefäße.

Ka Kathode aus Pt, An Anode aus Pb,

G Galvanometer, $R$ magnetischer Rührer.

hierzu die schematische Skizze, Abb. 2): Gefäß A, welches die anaerobe Kultur enthält, wird unter sterilen Kautelen mit dem Gaseinleitungsrohr e an den nach $\mathrm{H}$ aber gereinigten Stickstoff angeschlossen. Der Dreiweghahn g befindet sich in Stellung 2, so daß der Gasstrom durch

* Das gleiche Verfahren wird neuerdings auch in der elektrochemischen Oxygenometrie angewendet, um die in der Meßapparatur auftretenden Undichtigkeiten (Schnüffelstellen) zu lokalisieren. 
das Abzweigrohr $\mathrm{f}$ ins Freie tritt; dabei wird auch gleichzeitig die Hahndurchbohrung sauerstofffrei gespült. Alsdann schaltet man die Dreiweghähne g und $l$ von $\mathrm{Ge}-$ fäß A und die Dreiweghähne o und s von Gefäß B auf Stellung 1 und verbindet weiterhin das Einleitungsrohr $\mathrm{m}$ von Gefäß B, in welchem sich das frische Substrat befindet, mit dem Ableitungsrohr i von Gefäß A. Hiernach wird die Gesamtapparatur mit Haberschem Reinstickstoff bis zur vollständigen Desoxygenisierung durchgast.

Bei der darauffolgenden Beimpfung wird am Gefäß A der Dreiweghahn g auf Stellung 2 und der Dreiweghahn $l$ auf Stellung 4 geschaltet, während der Dreiweghahn s des Gefäßes B in Stellung 3 gebracht wird. Nunmehr neigt man das Gefäß A so weit zur Seite, daß sich der

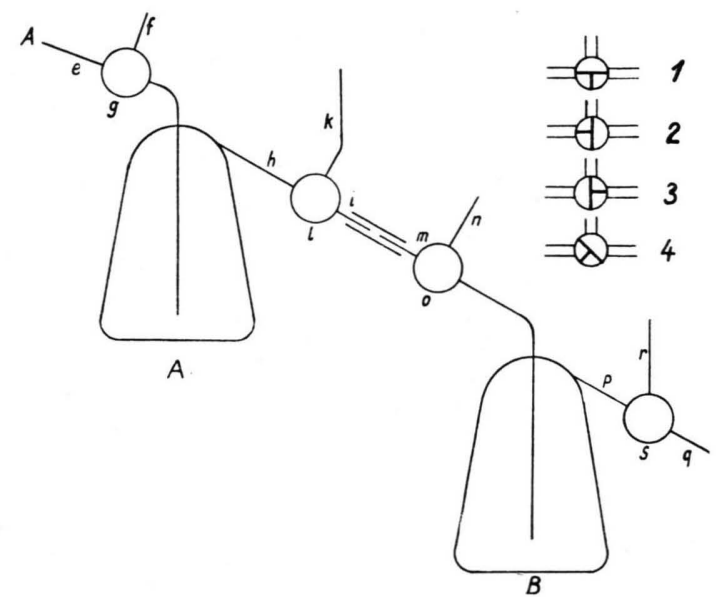

Abb. 2. Schematische Darstellung der Systematik des Arbeitsganges.

Schenkel h, der als Mensur ausgebildet ist, mit der Organismensuspension füllt. Wird jetzt Gefäß A wieder in die Normallage zurückgenommen, so verbleibt im Schenkel $\mathrm{h}$ die abgemessene Impfmenge. Diese wird, unter Schaltung aller Dreiweghähne auf Stellung 1, in das Gefäß B befördert. Schließlich bringt man die Dreiweghähne g und o (Gaseintritt beider Gefäße) in Stellung 2, die Dreiweghähne $l$ und s (Gasaustritt beider Gefäße) in Stellung 3, wonach eine neue anaerobe Passage ablaufen kann. Ob vollkommener Luftabschluß erreicht ist und während der gesamten Versuchsdauer aufrechterhalten bleibt, kann durch elektrochemische $\mathrm{O}_{2}$-Testung in Gefäß D laufend überwacht werden. Um jede Infektionsmöglichkeit zu vermeiden, werden die Wege, die nach außen führen, mit 70-proz. Alkohol desinfiziert und steril verschlossen. Bei absoluter $\mathrm{O}_{2}$-Abdichtung erfolgt der elektrochemische $\mathrm{O}_{2}$-Test in dem umgebenden Gasgefäß, und zwar an der Oberfläche der in ihm enthaltenen wässerigen Bodenflüssigkeit.

\section{Zellmaterial und Züchtungsansatz}

Zur Untersuchung gelangten die untergärigen Kulturhefen Stamm U, E und R, die obergärigen Kulturhefen $\mathrm{O}$ und W, die Spiritushefen Stamm XII und M, die Backhefen Stamm Tpl 152 und Lft 73 sowie die aus der Natur isolierten Torulazeen Stamm S und H. Die beiden reinkultivierten Wildhefenstämme besaßen ursprünglich kein Gärvermögen; sie wurden teils als nicht-gärende Organismen, teils nach zymagener Umzüchtung in das Versuchsprogramm einbezogen. Vor den entscheidenden Züchtungsansätzen erfolgte in jedem Falle die Herführung der dafür vorgesehenen Reinzuchtstämme bis zur Entfaltung ihrer optimalen Stoffwechselkräfte, so daß stets nur ein biologisch einwandfreies vitales Zellmaterial zur Verwendung kam. Mit Hilfe unserer Spezialapparaturen* waren wir in der Lage, vollkommen infektionsfrei zu manipulieren; außerdem führten wir noch die üblichen biologischen Kontrollen durch.

Als Nährsubstrat diente 10-proz. Würze $\left(p_{\mathrm{H}} 5,50\right.$ bis $5,60)$. Die Züchtungstemperatur betrug $20^{\circ}$. Die Zellenzahl, umgerechnet auf Zellen $/ \mathrm{mm}^{3}$, wurde mit Hilfe der Zeiß-Thomaschen Zählkammer ermittelt. Jede Passage führten wir so durch, daß wir auf die jeweilige Warmzüchtungsperiode eine mehrtägige Kaltlagerung zur Ausreifung der Juvenilzellen folgen ließen. Verschiedentlich wurde auch die entwickelte $\mathrm{CO}_{2}$-Menge quantitativ bestimmt.

$$
\begin{gathered}
\text { Anoxybiotische } \\
\text { Proliferationsversuche unter } \\
\text { normalisierten physiologischen } \\
\text { Bedingungen }
\end{gathered}
$$

Bei erneuter Durchführung der anaeroben Züchtungsversuche gingen wir von der anfänglich angewandten Eudiometrie ab und ersetzten sie, wie bereits beschrieben, durch das kürzlich von uns entwikkelte oxygenometrische Kombinationsverfahren, das nicht an die Verwendung von Quecksilber gebunden ist und außerdem alle biologischen Vorbedingungen restlos erfüllt. Auch bei strengster Wahrung des physiologischen Prinzips bestätigte sich generell der schon früher von uns erhobene Befund ${ }^{6}$, daß die Kulturhefen im anaeroben Substrat noch über eine längere Reihe von Passagen vermehrungsfähig bleiben, dabei aber ihr Proliferationsvermögen von Generation zu Generation successiv einbüßen, bis sie schließlich gänzlich zu wachsen aufhören. Im Zustand der Anabiose stirbt die Hefezelle jedoch nicht ab, sondern behält die Fähigkeit bei, nach aerober Reaktivierung völlig unbeschadet wieder wie vordem zu atmen, zu sprossen und zu gären. Selbst bei monatelanger Kaltlagerung unter Stickstoff konnte keine Abschwächung der Stoffwechselkräfte wahrgenommen werden, was den Gedanken nahelegt, daß die anoxybiotisch hervorgerufene Anabiose - im Vergleich zur hauptsächlich aerob erfolgenden Sporulation - eine Art von anaerober Dauerform der Zelle darstellt.

* Flammbier-Apparat bzw. Steril-Manipulator.

6 F. Wind is ch, Ergebn. Enzymforschg. 2, 169 [1933]. 
Aus Abb. 3 ist zu entnehmen, daß die Vermehrungsfähigkeit bei $\mathrm{O}_{2}$-freier Züchtung schon in der ersten Passage gegenüber dem oxybiotischen Vergleichsansatz beträchtlich abgenommen hat. Dennoch bleibt das Proliferationsvermögen - allerdings mit deutlich absinkender Tendenz — über mehrere Passagen erhalten; erst allmählich tritt vollständiger Wachstumsstillstand (Anabiose) ein. Die gleiche Veranlagung, jedoch mit graduellen Unterschieden, besitzen ausnahmslos alle von uns in dieser Richtung untersuchten Kulturhefen. beizubehalten (Abb. 4). Interessant ist hierbei die mikroskopische Beobachtung, daß bei fortschreitender Abschwächung der Wuchskraft die Tochterzellen teilweise nur noch unvollständig ausgebildet sind und von der Mutterzelle nicht mehr abgestoßen werden.

Aus dem anoxybiotischen Sproßvermögen der aerob gärfähig gewordenen Torulazeen läßt sich — gegenüber ihrer ursprünglich nur aeroben Wachstumsfähigkeit - noch deutlicher als bei den Kulturhefen erkennen, daß mit der zymagenen Hochzüchtung zugleich auch die Befähigung zum Speichern von

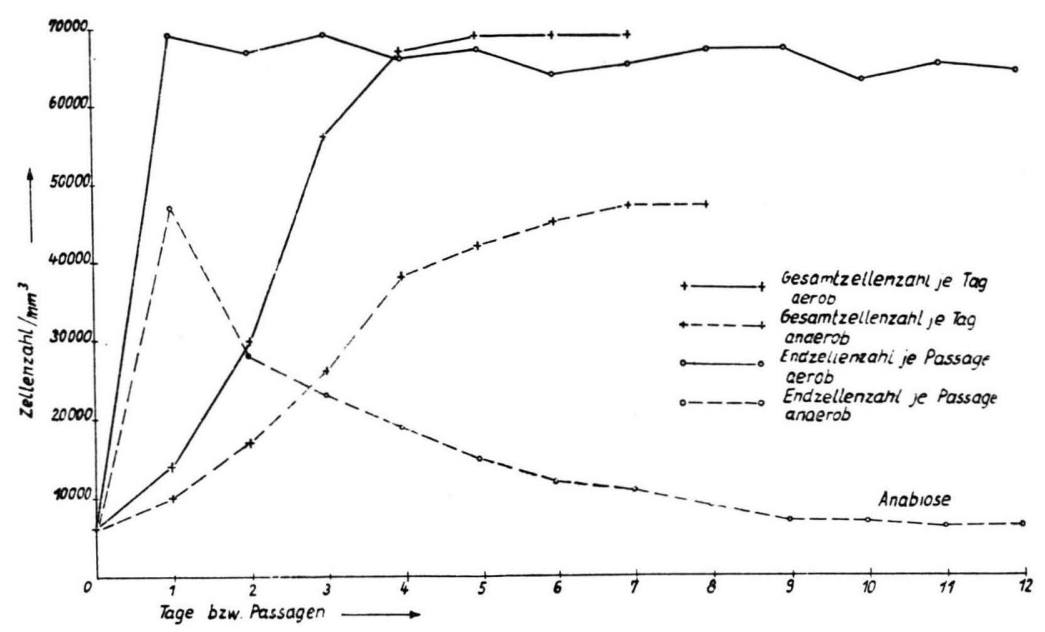

Abb. 3. Anaerobe und aerobe Zellvermehrung von untergäriger Kulturhefe Stamm R.

Besonders aufschlußreich waren die mit Torulazeen durchgeführten anoxybiotischen Züchtungsversuche. Aus der Natur isoliert, sind die Torulopsisarten meistens nicht gärfähig. Durch zymagene Hochzüchtung ${ }^{7}$ lassen sie sich aber verhältnismäßig leicht in aerob gärende Organismen ${ }^{8}$ umwandeln.

Unsere Untersuchungen haben ergeben, daß die nicht-gärenden Torulazeen außerstande sind, sich anoxybiotisch fortzupflanzen. Demnach kann auch die zymagene Umzüchtung nicht vollständig anaerob erfolgen, was empirisch bekannt, aber im vorliegenden Zusammenhang um so beachtlicher ist. Sind dagegen die ursprünglich nicht-gärenden Wildhefen durch $\mathrm{O}_{2}$ arme Hochzüchtung im irreversiblen Proze $\aleph^{9}$ zu aerob spaltbefähigten Alkoholbildnern umgewandelt, so vermögen sie genau wie die Kulturhefen anaerob zu sprossen und diese Fähigkeit, wenn auch mit stark absinkender Wirkung, noch über einzelne Passagen

7 F. H a y d u c k u. H. H a e h n, Biochem. Z. 128, 568 [1922].

8 F. W in d i s c h, Naturwiss. 34, 190 [1947].
Wachstumsenergie entwickelt wird. Nicht unwesentlich ist hiervon die $\mathrm{O}_{2}$-Bilanz der Zellvermehrung abhängig, die nach der bisherigen Konzeption bei unterwertiger Sauerstoffversorgung nicht aufgeht. Übrigens vertreten neuerdings auch Soskin und Levine ${ }^{10}$ die Ansicht, daß im Muskel eine Energievorratsbildung stattfindet; nach dem von ihnen entworfenen Schema des Energiewechsels stellt Phosphokreatin das energiespeichernde, ATP das energieübertragende System dar. Ähnliche Systeme müssen nach unseren Feststellungen auch in der Hefezelle vorliegen; als Depotstoff könnte hierbei das analoge pflanzliche Phosphagen, die Argininphosphorsäure, in Betracht kommen ${ }^{11}$.

Die anaerob gewachsenen Organismen zeichnen sich durch ein stärkeres Gärvermögen aus als die

9 Gemeinschaftsarbeit von F. W in d i s c h, D. S t i e r a nd sowie von H. H a ehn, Protoplasma 1953 (im Druck).

10 S. S o skin u. R. L e vine, Carbohydrate Metabolism. Chicago und London 1946.

$11 \mathrm{H}$. H a e h n, Biochemie der Gärungen. Berlin 1952. 
aerob gezüchteten, wie aus Abb. 5 hervorgeht. $\mathrm{Zu}$ dem gleichen Ergebnis sind wir bereits bei früheren Untersuchungen ${ }^{12}$ gelangt.

In einer kürzlich erschienenen Arbeit berichtet $\mathrm{S} \mathrm{ch} \mathrm{m} \mathrm{i} \mathrm{d}{ }^{13}$ darüber, daß bei der Atmungshemmung durch $m / 500$ - bis $m / 1000$-Blausäure auch die Zellvermehrung ,schlagartig aufgehoben“ wird, während die Gärung stark ansteigt. Mit Entfernung der Blausäure gehen nach $\mathrm{Schmid}$ alle diese Wirkungen, entsprechend dem $\mathrm{M}$ e y e r h of - Quotienten ${ }^{14}$, rasch und vollständig zurück. Demgegenüber stellten wir fest, daß die durch anaerobe Sprossung erhöhte

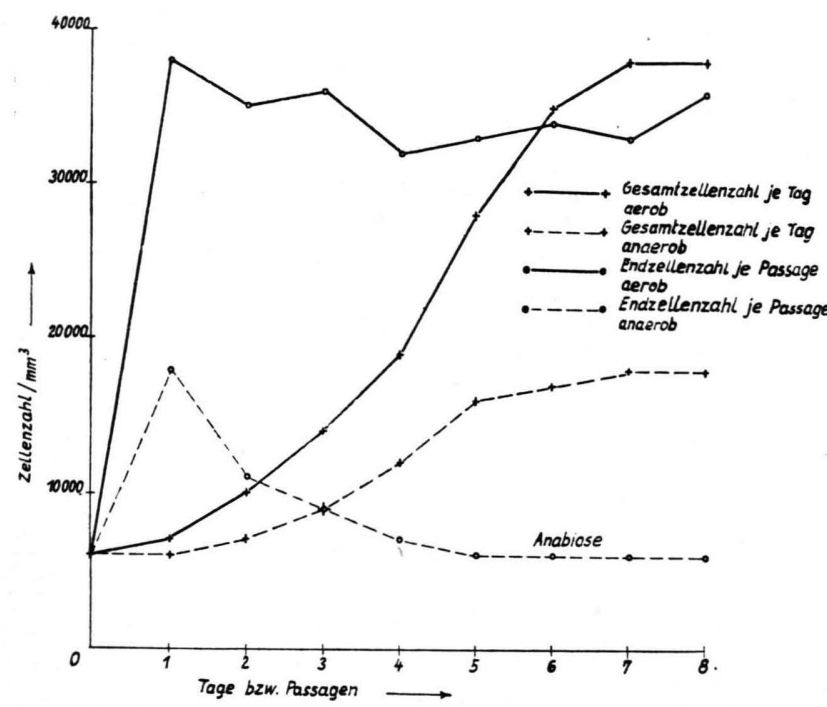

Abb. 4. Anaerobe und aerobe Zellvermehrung von hochgezüchteter Torulopsis Stamm S.

Gärkraft der Hefe, bei nicht-vermehrungsfähiger Aussaat, in der Oxybiose persistiert, woraus wir in Verbindung mit früheren Ergebnissen ${ }^{15}$ schließen möchten, daß es sich hierbei um eine vegetative Umwandlung der Zelle handeln muß. Beide Wirkungen, die der Blausäure, welche reversibel die Eisenkatalyse in der Zelle ausschaltet ${ }^{16}$, und die der Anoxybiose, welche anscheinend von vegetativem Einfluß ist, sind physiologisch verschieden und können nicht ohne weiteres zueinander in Parallele gesetzt werden. Mit der Schlußfolgerung S chmids, „daß die Zellvermehrung der Hefe trotz ihrer anaeroben Spezialisierung einen obligat aeroben Vorgang darstellt", stimmen unsere Versuche zwar im Endergeb-

12 F. W ind is ch, Ergebn. Enzymforschg. 2, 169 [1933], und zwar S. 173/174.

1s W. S c h m i d, Z. Naturforschg. 7 b, 217 [1952]. nis überein, aber nicht in der Deduktion. Anscheinend ist es $\mathrm{S} \mathrm{chmid}$ bei der Art seiner Versuchsanordnung und infolge zu kurzer Beobachtungszeiten entgangen, daß die Gärhefen auch in der Anoxybiose partiell zu sprossen vermögen. Erst durch die Aufklärung dieses scheinbar widersprechenden Phänomens in der von uns deduzierten Weise rechtfertigt

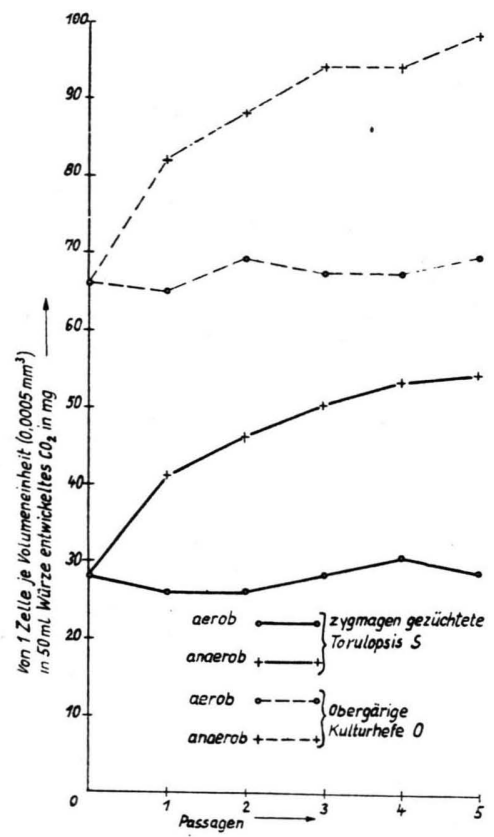

Abb. 5. Gärungsintensität von obergäriger Kulturhefe Stamm O und hochgezüchteter Torulopsis Stamm S, vergleichend gemessen nach anaerober und aerober Kultivierung.

sich die von $\mathrm{S}$ ch mid aufgestellte These, daß selbst die anoxybiotisch „stark erhöhte Gärung energetisch nicht ausreicht, um eine praktisch in Betracht kommende Zellvermehrung zu unterhalten“. Übereinstimmend hiermit haben unsere Versuche generell ergeben, daß die Kontinuität des Hefewachstums obligat aerob bedingt ist.

In Anwendung auf die biologische Krebsforschung (Gemeinschaftsarbeit mit Prof. Graffi) sind wir dazu übergegangen, nach dem gleichen methodischen Prinzip die Wachstumsfähigkeit der Krebszellen unter anaeroben Bedingungen bzw. bei variierten $\mathrm{O}_{2}$ Partialdrucken zu untersuchen, um auf entsprechende Weise - in Analogie zur Hefegärung - über die

14 O. M e y e r h of , Biochem. Z. 162, 43 [1925].

15 F. W in d is c h, Naturwiss. 34, 190 [1947].

16 O. W a r bu r g, Biochem. Z. 152, 479 [1924]. 


\begin{tabular}{|c|c|c|}
\hline \multirow[b]{2}{*}{ Belüftungsart } & \multicolumn{2}{|c|}{ Zellenzahl $/ \mathrm{mm}^{3}$} \\
\hline & $\begin{array}{c}\text { Im Anschluß } \\
\text { an die anaerobe } \\
\text { Passagen- } \\
\text { züchtung } \\
\text { 12. Passage } \\
2600 \\
\text { (Aussaatmenge) }\end{array}$ & $\begin{array}{c}\text { Nach } \\
\text { ca. 6-monatiger } \\
\text { Kaltlagerung } \\
\\
2000 \\
\text { (Aussaatmenge) }\end{array}$ \\
\hline $\begin{array}{ll}\text { Initialbelüftung } & \text { I } \\
\text { Initialbelüftung } & \text { II } \\
\text { Initialbelüftung } & \text { III }\end{array}$ & $\begin{array}{l}\text { unverändert } \\
\text { unverändert } \\
\text { unverändert }\end{array}$ & $\begin{array}{l}\text { unverändert } \\
\text { unverändert } \\
\text { unverändert }\end{array}$ \\
\hline $\begin{array}{l}\text { 1. Belüftungsphase } \\
\text { 2. Belüftungsphase } \\
\text { 3. Belüftungsphase } \\
\text { 4. Belüftungsphase } \\
\text { 5. Belüftungsphase } \\
\text { 6. Belüftungsphase } \\
\text { 7. Belüftungsphase } \\
\text { 8. Belüftungsphase }\end{array}$ & $\begin{array}{r}7600 \\
15200 \\
32000 \\
38600 \\
40200 \\
41800 \\
42200 \\
42000\end{array}$ & $\begin{array}{r}8600 \\
14400 \\
29200 \\
40600 \\
42800 \\
44600 \\
43800 \\
44200\end{array}$ \\
\hline $\begin{array}{l}\text { Kontinuierliche } \\
\text { Belüftung }\end{array}$ & 69000 & 71600 \\
\hline
\end{tabular}

Tab. 1. Aerobe Wachstumsreaktivierung der anabiotischen Unterhefe Stamm U.

biosynthetische Potenz der Krebsglykolyse Aufschluß zu erhalten.

\section{Wirkungsmechanismus der aeroben Wachstumsreaktivierung}

Die Kulturhefen im anabiotischen Zustand sowie die nicht-gärenden und anaerob nicht-vermehrungsfähigen Torulazeen geben ein geeignetes Ausgangsmaterial ab, um der noch unbeantworteten biologischen Frage näherzukommen, nach welchem Modus die aerobe Wachstumsreaktivierung vor sich geht.

Methodisch führten wir die periodisierten Aerationsversuche in der Weise durch, daß wir nach Verdrängung des Stickstoffs zunächst kurzfristige Luftstöße (Initialeffekt) durch das anaerobe Kulturgut sandten. Wenn diese nicht ausreichten, um das Wachstum zu reaktivieren, gingen wir zur Phasenbelüftung über, in deren Grenzen es dem Sauerstoff möglich ist, auf die anaerobe Zellkultur einzuwirken. Jede einzelne Belüftungsphase fand ihren Abschluß durch kräftig einsetzende $\mathrm{N}_{2}$-Vergasung bei erhöhter Durchströmungsgeschwindigkeit (etwa $5 \mathrm{l} /$ Stde.). Die nächste Applikation mit Luft nahmen wir stets erst dann wieder vor, wenn keine Zellvermehrung mehr festzustellen war. Zur Kontrolle der maximalen Wachstumsfähigkeit wurden in allen Versuchsreihen je zwei der Züchtungsansätze kontinuierlich belüftet.

Als Beispiel der aeroben Wachstumsreaktivierung

\begin{tabular}{|c|c|c|}
\hline \multirow[b]{2}{*}{ Belüftungsart } & \multicolumn{2}{|c|}{ Zellenzahl $/ \mathrm{mm}^{3}$} \\
\hline & $\begin{array}{l}\text { Unmittelbar } \\
\text { nach der } \\
\text { Lüftungs- } \\
\text { züchtung } \\
4800 \\
\text { (Aussaatmenge) }\end{array}$ & $\begin{array}{c}\text { Nach } \\
\text { 3-wöchiger } \\
\text { anaerober } \\
\text { Kaltlagerung } \\
5600 \\
\text { (Aussaatmenge) }\end{array}$ \\
\hline $\begin{array}{l}\text { Initialbelüftung I } \\
\text { Initialbelüftung II } \\
\text { Initialbelüftung III }\end{array}$ & $\begin{array}{l}\text { unverändert } \\
\text { unverändert } \\
\text { unverändert }\end{array}$ & $\begin{array}{l}\text { unverändert } \\
\text { unverändert } \\
\text { unverändert }\end{array}$ \\
\hline $\begin{array}{l}\text { 1. Belüftungsphase } \\
\text { 2. Belüftungsphase } \\
\text { 3. Belüftungsphase } \\
\text { 4. Belüftungsphase } \\
\text { 5. Belüftungsphase } \\
\text { 6. Belüftungsphase } \\
\text { 7. Belüftungsphase } \\
\text { 8. Belüftungsphase } \\
\text { 9. Belüftungsphase } \\
\text { 10. Belüftungsphase }\end{array}$ & $\begin{array}{r}16400 \\
32000 \\
80800 \\
209600 \\
429600 \\
526000 \\
584800 \\
576400 \\
558400 \\
560800\end{array}$ & $\begin{array}{r}20800 \\
40400 \\
97600 \\
198800 \\
398800 \\
571200 \\
599200 \\
606400 \\
612800 \\
620000\end{array}$ \\
\hline $\begin{array}{l}\text { Kontinuierliche } \\
\text { Belüftung }\end{array}$ & 1020000 & 995000 \\
\hline
\end{tabular}

Tab. 2. Wachstumsverlauf von nicht-gärender und anaerob nicht-vermehrungsfähiger Torulopsis Stamm H bei Initialund Phasenbelüftung.

nach anoxybiotisch hervorgerufener Anabiose sind in Tab. 1 die mit Unterhefe Stamm U von uns erzielten Ergebnisse aufgeführt, welche einem vielseitigen Untersuchungsmaterial entnommen sind; es besteht mit denen der anderen von uns einbezogenen Gärhefen eine prinzipielle Übereinstimmung. Das verwendete Impfgut, welches in Parallelversuchen einmal sofort nach der letzten anabiotischen Passage und zweitens erst nach mehrmonatiger Kaltlagerung zur aeroben Reaktivierung angesetzt wurde, enthielt in allen überprüften Fällen nur einen geringen Prozentsatz an abgestorbenen Zellen und gab im Kontakt mit Luft, besonders auch nach den ausgiebigen Ruhezeiten, eine unverminderte Vitalität zu erkennen.

Wie aus der tabellarischen Zusammenstellung zu entnehmen ist, findet bei der Einwirkung von kurzfristigen Luftstößen auf die anabiotische Hefe kein Wachstum statt, was sich auch bei allen Reaktivierungsversuchen mit anderen Kulturhefestämmen ausnahmslos bestätigte. Erst beim Übergang zur Phasenbelüftung, die nach vorausgegangener Testung auf je 60 Min. bemessen wurde, setzt die Zellvermehrung ein und schreitet, entsprechend der periodischen Belüftungsvornahme, successiv bis zu einem relativen Wachstumsmaximum fort, das allerdings, wie kontrollmäßig ermittelt wurde, unter dem der kontinuierlich belüfteten Ansätze liegt. Bei Überimpfung 
der mehrfach periodisch belüfteten, aber noch nicht endvermehrten anabiotischen Hefen in frische anaerobe Nährlösung stagniert sogleich wieder das Wachstum, woraus entnommen werden kann, daß im Reaktivierungsstadium noch keine oder zumindest keine ausreichende Energiespeicherung in der Zelle stattgefunden hat.

Der gleiche aerobe Wirkungsmechanismus tritt in noch ausgeprägterer Form bei nicht-gärenden und anaerob nicht-vermehrungsfähigen Torulazeen in Erscheinung. Als Beispiel hierfür sind in Tab. 2 die Wachstumsverhältnisse von Torulopsis Stamm H angeführt, die in gleicher Richtung mit den anderen von uns angestellten, aber in sich eigenwertig verlaufenden Kultivierungsversuchen liegen. Bei der biologischen Vorprüfung des Zellmaterials stellten wir fest, daß die aus der Natur isolierten Torulazeen - im Gegensatz zu den obligat aeroben Essigbildnern ${ }^{17}$ eine längere anaerobe Kaltlagerung ohne merkliche Beeinträchtigung vertragen. Aus den tabellarisch wiedergegebenen Zahlreihen ist $\mathrm{zu}$ ersehen, daß kurze Aerationsstöße nicht imstande sind, das anoxybiotisch sistierte Zellwachstum mit initialer Wirkung in Gang zu bringen, weder im unmittelbaren Anschluß an die Lüftungszüchtung noch nach einer längeren anaeroben Lagerzeit; mikroskopisch war ebenfalls kein Anzeichen von Proliferation erkennbar. Erst beim Übergang zur Phasenbelüftung - von je 120 Min., entspr. der vorausgegangenen Testung setzt successiv die Zellvermehrung ein und verläuft nach der gle:chen Gesetzmäßigkeit wie bei den anabiotischen Gärhefen. Das bei den letzteren eruierte Wachstumsprinzip gilt also in gleicher Weise auch für die aus der Natur isolierten Torulopsishefen, bei denen zum Teil wiederum wesentlich andere biologische Umstände in Betracht zu ziehen sind. Weitere experimentelle Belege und Einzelheiten, die über den Rahmen dieser Veröffentlichung hinausgehen, werden nachfolgend in der Biochemischen Zeitschrift gebracht.

Auf Grund unserer eindeutigen Versuchsergebnisse

17 O. Hromatka, H. Ebner u. Chr. Csokli c h, Enzymol. Acta Biocatalyt. 15, 134 [1951]. kann die Frage nach dem Mcdus der Sauerstoffwirkung bei anoxybiotisch hervorgerufener Wachstumssistierung dahingehend beantwortet werden, daß der aerobe Reaktivierungsproze $ß$ nicht durch einen initialen $\mathrm{O}_{2}$-Effekt ausgelöst wird, sondern zeitlich abhängig ist von dem in Reaktion tretenden Sauerstoff, der seinerseits, wie bereits früher von uns festgestellt ${ }^{18}$, in quantitativer Relation zur proliferierenden Zellmasse steht. Die Aufklärung dieser generativen Gesetzmäßigkeit war erst möglich, nachdem wir erkannt hatten, daß die stöchiometrische Proportionalität zwischen $\mathrm{O}_{2}$-Wirkung und Zellwachstum nur dann biologisch exakt zu statuieren ist, wenn dabei von Zellen ausgegangen wird, die keine Energiereserve enthalten.

Die anabiotischen Gärhefen, deren natives Energiedepot sich in den $\mathrm{O}_{2}$-freien Züchtungspassagen restlos aufbraucht, verfügen im aeroben Reaktivierungsstadium, wie bereits vorher ausgeführt, noch nicht wieder über den erforderlichen Energievorrat, um sich anoxybiotisch fortpflanzen zu können. Demgegenüber entwickeln die aus der Natur isolierten Torulazeen, die ausschließlich atmen und anaerob nicht zu wachsen vermögen, im Verlauf ihrer zymagenen Umzüchtung die Eigenschaft, Oxydationsenergie zu speichern, mittels derer sie befähigt sind, in begrenztem Umfange sauerstofffrei zu sprossen.

Die Bildung von Reserveenergie in den Gärhefen scheint demnach dem biologischen Zweck zu dienen, bestimmten fakultativen Anaerobiern die Möglichkeit zu geben, selbst noch in Perioden der Sauerstoffnot oder vollständigen Anoxybiose partiell zu proliferieren. In Analogie hierzu werden uns die gemeinsam mit der Abt. Biologische Krebsforschung aufgenommenen Untersuchungen Kenntnis darüber vermitteln, ob auch die Krebszellen über eine oxydativ gebildete Energiereserve verfügen, deren Nachweis zur Aufklärung der widersprechenden Angaben über das anaerobe Krebswachstum ${ }^{19}$ in gewissem Sinne beitragen dürfte.

18 F. W in dis ch, Biochem. Z. 246, 332 [1932], und zwar S. 355 u. S. 360.

19 Zusammenfassende Darstellung bei E. K n a k e: Z. Krebsforschg. 52, 269 [1942]. 